\title{
Measurement and simulation for a complementary imaging with the neutron and X-ray beams
}

\author{
Kaoru Y. Hara ${ }^{1, a}$, Hirotaka Sato ${ }^{1}$, Takashi Kamiyama ${ }^{1}$, and Takenao Shinohara ${ }^{2}$ \\ ${ }^{1}$ Faculty of Engineering, Hokkaido University, Sapporo 060-8628, Japan \\ 2 J-PARC Center, Japan Atomic Energy Agency, Ibaraki-ken 319-1195, Japan
}

\begin{abstract}
By using a composite source system, we measured radiographs of the thermal neutron and keV $\mathrm{X}$-ray in the $45-\mathrm{MeV}$ electron linear accelerator facility at Hokkaido University. The source system provides the alternative beam of neutron and X-ray by switching the production target onto the electron beam axis. In the measurement to demonstrate a complementary imaging, the detector based on a vacuum-tube type neutron color image intensifier was applied to the both beams for dual-purpose. On the other hand, for reducing background in a neutron transmission spectrum, test measurements using a gadolinium-type neutron grid were performed with a cold neutron source at Hokkaido University. In addition, the simulations of the neutron and X-ray transmissions for various substances were performed using the PHITS code. A data analysis procedure for estimating the substance of sample was investigated through the simulations.
\end{abstract}

\section{Introduction}

A complementary imaging using a difference between the penetration abilities of neutron and X-ray is expected to enhance information which is obtained from an individual $\mathrm{X}$-ray or neutron image such as materials and structures of an object. For the complementary imaging, we had installed a composite source system of thermal neutron and $\mathrm{keV} \mathrm{X}$-ray in the $45-\mathrm{MeV}$ electron linear accelerator facility at Hokkaido University [1,2]. The source system is an accelerator-driven and provides the alternative beam of neutron and X-ray by switching the production target onto the electron beam axis. In addition, we have developed the measurement method of the images for the both beams by using a two-dimensional neutron detector [3]. In order to demonstrate the good point of a combination of the composite source system and the dual-use detector, transmission images for a sample were measured using the neutron and X-ray beams. Additionally, test measurements using a neutron grid were performed for reducing background in a neutron transmission spectrum. On the other hand, a data analysis procedure for estimating the substance of sample was investigated using the MonteCarlo simulation code PHITS [4]. In this paper, the recent measurements and simulations for the complementary imaging with the neutron and X-ray beams are reported.

\section{Experimental}

\subsection{Composite source system}

As shown in Fig. 1, the composite source system consists of two production targets, a moderator, reflectors, radiation shields, and an up-and-down stage [1,2]. To produce a neutron or X-ray beam, the alternative target is set on the axis of electron beam by remotely moving the up-and-

a e-mail: hara.kaoru@eng.hokudai.ac.jp down stage. The horizontal cross-sectional view of the neutron and X-ray production targets are shown in the insets (a) and (b) of Fig. 1, respectively. In the neutron production, the $34-\mathrm{MeV}$ electron beam is irradiated to an assembled target that is composed of a tungsten disc, lead blocks, water-cooling channels. The bremsstrahlung photons are produced by the electron irradiation and induce the neutrons through the photonuclear reaction of lead. The neutrons are slowed down with the polyethylene moderator which are surrounded by graphite reflectors. The moderated neutrons, which are emitted into the perpendicular direction with respect to the electron beam axis, are used as the neutron beam. In the X-ray production, the $9-\mathrm{MeV}$ electron beam is irradiated to a copper plate target to produce bremsstrahlung photons. The bremsstrahlung photons which are emitted into the perpendicular direction with respect to the electron beam axis are used as the X-ray beam. The energy distributions of the neutron and X-ray beams are described in Sect. 3.1.

\subsection{Detector for both neutron and X-ray}

In a measurement for the complementary imaging, a detector based on a vacuum-tube type neutron color image intensifier (NCII; TOSHIBA, Ultimage ${ }^{\mathrm{TM}}-\mathrm{n} \gamma-04$ ) [5] has been applied to the both neutron and X-ray beams. The schematic view of the detector is shown in Fig. 2 A $\mathrm{Pb}$ sheet, a $\mathrm{Gd}_{2} \mathrm{O}_{3}$ layer evaporated on an $\mathrm{Al}$ sheet, a CsI scintillator, and a photocathode are mounted on the input window of NCII, where the diameter of input window is 4 inch. For the efficient neutron detection, the incident neutrons are dominantly converted to internal conversion electrons through the neutron capture reaction of gadolinium. When the conversion electrons are detected in the CsI scintillator, fluorescence photons are yielded, where the thickness of CsI, which is optimized for a neutron imaging, is $50 \mu \mathrm{m}$. The NCII are applied not only 


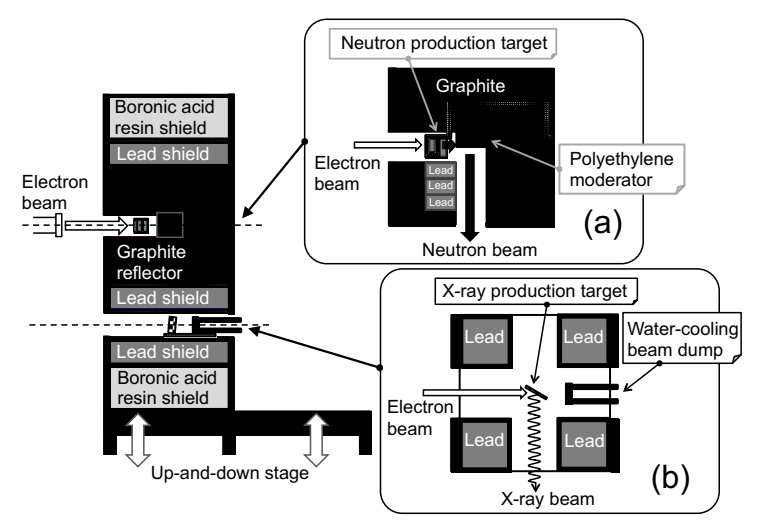

Figure 1. Composite source system in the electron linac facility of Hokkaido University.

to the neutron imaging but also to the X-ray imaging because the CsI scintillator can detect the X-rays while it has not high detection efficiencies for the keV X-rays. The succeeding process after the fluorescence is same for either incident beam. These fluorescence photons produce photoelectrons in the photocathode. The photoelectrons are converged by the electrodes of vacuum tube in order to project on a output screen $\left(\mathrm{Y}_{2} \mathrm{O}_{2} \mathrm{~S}\right.$ :Eu phosphor). The visible photons which are emitted from the output screen are collected to an image recorder through an optical lens and mirror.

For the NCII, we have used a digital photographic camera (CANON, EOS 5D Mark II) or a high-speed camera (DITECT, HAS-D3) as the image recorder. Neutron and X-ray transmission images, which are radiographs, can be measured using the NCII combined with the digital camera [3] as described in Sect. 2.3. Although the differences of substances and the shapes are estimated from the radiographs, the types of substances are not well identified because our digital camera system records an image having integrated brightness vales over an exposure time. For measuring transmission images with the neutron time-of-flight (TOF) method, a development is ongoing using the NCII combined with the high-speed camera. By applying the TOF method, further information about substances of a sample would be obtained since neutron transmissions depend on incident neutron energies and the substances. In addition, a background component due to a $\gamma$-flash [6], which is contaminated in a neutron pulse at the initial time of TOF, would be removed from the neutron transmission image by gating the TOF range. The details of measurements with the high-speed camera system will be reported in a next work.

\subsection{Complementary imaging}

The transmission images for neutron and X-ray were measured by using the composite source system without change of a sample and detector position. The measurements for the neutron and X-ray imaging were performed with the detector which is combined the NCII and the digital camera. The experimental setup is shown in Fig. 2. In order to adequately suppress the $\gamma$-flash that was contaminated in the neutron beam, lead blocks of 5-cm thickness were located on the front of a sample and the detector when the neutron imaging. The sample was a light emitting diode (LED) flashlight involving a nickel-metal

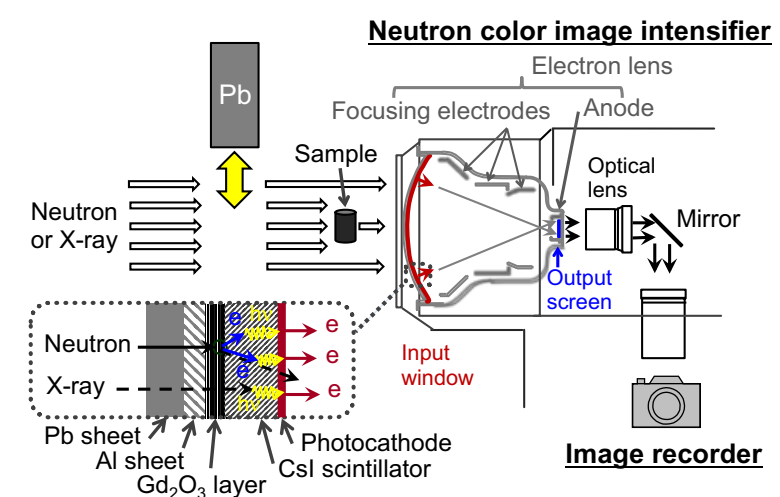

Figure 2. Experimental setup of the neutron and X-ray detector system.

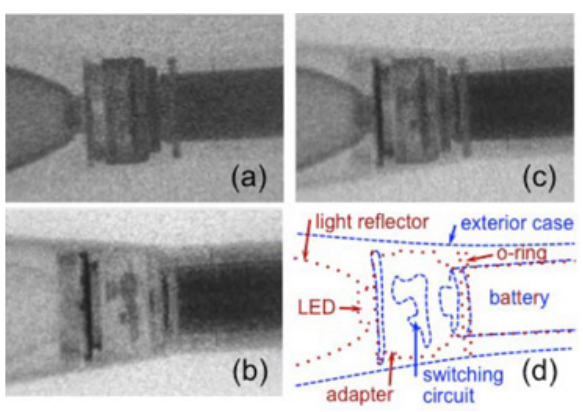

Figure 3. Transmission images of the LED flashlight measured with the neutron (a) and X-ray beam (b). The transmissions of (a) and (b) at each pixel are summed (c). The sketch represents the interior structure (d).

hydrogen $(\mathrm{NiMH})$ rechargeable battery. The distance was about $7 \mathrm{~m}$ from the polyethylene moderator to the sample (or detector). The measured images for the neutron and X-ray beam are shown in Fig. 3(a) and (b), respectively. The view area was about $5 \mathrm{~cm} \times 4 \mathrm{~cm}$ and the number of effective pixels was $5616 \times 3744$. The image was obtained by calculating the ratio of the brightnesses of images, which were measured with and without the sample, at each pixel. Here, we call the ratio "transmission ratio". The each measurement time was several minutes. After the data of image were treated as the CANON RAW (CR2) format using the DCRAW reader plugged in the ImageJ program [7], these were converted from CR2 to JPEG. As shown in Fig. 3(a) and (b), the interior structure for the same sample is non-destructively visualized while the profiles are different according to the beam type. Since the images were measured without any change of the setup of sample and detector, it allowed us to directly compare and complement each other. To demonstrate the advantage, we therefore derived an image by adding the transmission ratio of neutron (a) to X-ray (b) at each pixel. The contrast of image was enhanced as shown in Fig. 3(c). The shapes of the light reflector, LED, switching circuit adapter, o-ring, and NiMH battery are sighted inside the exterior case. As an eye guide, a sketch for the interior structure is shown in Fig. 3(d).

\subsection{Neutron grid}

In this section, we report about the neutron grid method to improve neutron transmission measurements apart from $\mathrm{X}$-ray transmission measurements. One of background 


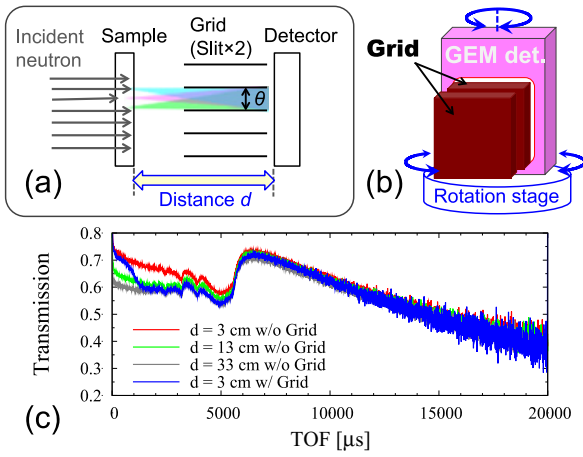

Figure 4. Conceptual diagram of the grid method (a). In the test measurements, the Gd-type neutron grid and the GEM detector were used (b). The neutron transmission spectra for the iron sample were measured with and without the grid (c).

components is a scattering neutron from a sample in neutron transmission measurements. The overestimation of the measured transmission arises from the detection of the scattered neutron [8]. To supress the background neutrons to enter the detector, we have planned to apply a neutron grid to the transmission measurement. The concept of the grid method is presented in Fig. 4(a), where a slit, which consists of many layers of neutron absorption and transparent materials, is just illustrated because the grid is a pair of crossing slits. The slit can restrict the solid angle of the scattered neutron. An alternative method to the grid is to properly keep a distance $d$ from the sample to the detector. An example of the dependence of $d$ on Bragg edge transmission spectra, which were calculated using the PHITS, was reported in Ref. [8]. However, the image degradation is caused by the beam divergence with increasing $d$.

Test measurements for supressing the background using a gadolinium-type neutron grid were performed on the beam line of cold neutron source at Hokkaido University [9]. The slit width, area, and depth of the grid were $0.15 \mathrm{~mm}, 10 \mathrm{~cm} \times 10 \mathrm{~cm}$, and $0.4 \mathrm{~cm}$, respectively. The flight path between the moderator and the detector was about $5 \mathrm{~m}$. The schematic view of the experimental setup is shown in Fig. 4(b). The neutron detector was used a gas electron multiplier (GEM) detector [10]. The grid was placed at the front of the GEM detector. In this test measurement, a rotational stage was only used to adjust the angle of the slit against to the incident neutron beam. The sample was an iron plate with the size of $10 \mathrm{~cm} \times$ $10 \mathrm{~cm} \times 0.5 \mathrm{~cm}^{\mathrm{t}}$. The neutron transmission spectra for the iron sample which were measured with and without the grid are shown in Fig. 4(c). In these spectra, the Braggedges are observed. The blue line shows the measurement with the grid at $d=3 \mathrm{~cm}$. The red, green, and gray lines show the measurements without the grid at $d=3,13$, and $33 \mathrm{~cm}$, respectively. For the measurements without the grid, the transmission in the TOF region below $5000 \mu \mathrm{s}$ increases with decreasing $d$. The difference in the fast neutron region is derived from the contamination of background neutrons. By contrast, in spite of $d=3 \mathrm{~cm}$, the transmission for the measurement with the grid (blue line) is consistent with the gray line in the TOF region above $1000 \mu$ s i.e. the neutron energy region below $0.2 \mathrm{eV}$. Below $0.2 \mathrm{eV}$, it is expected that the grid is useful for reducing the background due to the scattering neutron from

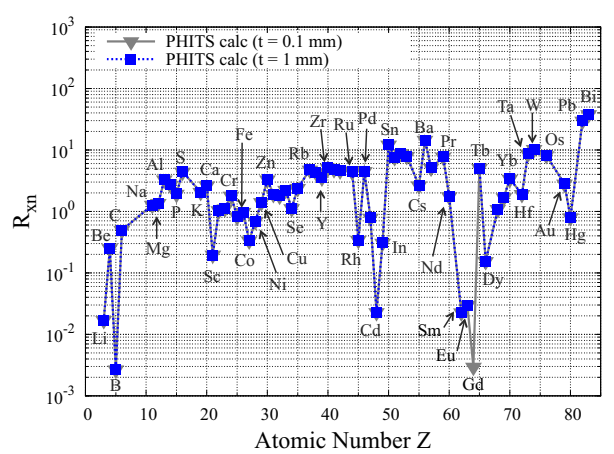

Figure 5. $R_{\mathrm{xn}}$ for various samples. The $R_{\mathrm{xn}}$ is obtained from the calculated transmissions of neutron and X-ray using Eq. (2).

the sample in the neutron transmission measurement. The usefulness of the grid would be same in principle with the measurements using another neutron detector for the neutron beam of composite source system.

\section{Simulation}

\subsection{Energy distributions of the neutron and X-ray beams}

The energy distributions of the neutron and X-ray beams were calculated with the PHITS code, where JENDL4.0 was used to the neutron cross section data [11]. The calculated energy distribution was presented in Ref. [2]. In the simulation for the neutron beam, an isotropic point source of neutrons was defined as an initial source because of the computation time although the actual neutron production was initiated from the electron beam.

The point source with the energy of the MaxwellBoltzmann distribution at $k T=1.3 \mathrm{MeV}$ was placed at the inside of $\mathrm{Pb}$ block. The neutrons, which were moderated by the polyethylene moderator $\left(12 \mathrm{~cm} \times 12 \mathrm{~cm} \times 5 \mathrm{~cm}^{\mathrm{t}}\right)$, were counted by a tally at a distance of $100 \mathrm{~cm}$ from the moderator. The exit aperture for the neutron beam is an area of $10 \mathrm{~cm} \times 10 \mathrm{~cm}$. The energies of neutrons widely ranged from $\mathrm{meV}$ to $\mathrm{MeV}$ and the average energy in the peak region of 2-200 meV was approximately $30 \mathrm{meV}$. On the other hand, in the simulation for the X-ray beam, the pencil-like electron beam with the diameter of $1 \mathrm{~cm}$ and the energy of $9 \mathrm{MeV}$ was defined as an initial source. The bremsstrahlung photons which were produced by the electron beam irradiation on the copper target $(7 \mathrm{~cm} \times$ $7 \mathrm{~cm} \times 0.4 \mathrm{~cm}^{\mathrm{t}}$ ) were counted by a tally at a distance of $100 \mathrm{~cm}$ from the target. The bremsstrahlung photons i.e. $\mathrm{X}$-rays had a continuous distribution below several MeV and the average energy in the region of $0.01-10 \mathrm{MeV}$ is approximately $200 \mathrm{keV}$.

\subsection{Transmissions for various samples}

As mentioned below, we have investigated with the PHITS code in order to adapt an idea into the data analysis of our complementary imaging. Two equations are expressed to explain the idea. First, a transmission for a sample with a thickness $t$ is defined by

$$
T r_{\mathrm{i}}=I_{\text {out }} / I_{\text {in }}=\exp \left(-\mu_{i} t\right) .
$$

The $I_{\text {in }}$ and $I_{\text {out }}$ are the intensities of the incident and transmitted beam, respectively. The $\mu_{\mathrm{i}}$ is the linear 
attenuation coefficient and its quantity depends on the beam type and the energy, where the subscript $i$ is replaced as the beam type i.e. $\mathrm{n}$ (neutron) or $\mathrm{x}$ (X-ray). For simplicity, the energies of neutron and X-ray are treated as monochromatic here. Second, the ratio of the $\log$ of $T r_{\mathrm{x}}$ to the $\log$ of $T r_{\mathrm{n}}$ is written by substituting Eq. (1) as

$$
R_{\mathrm{xn}}=\ln \left(T r_{\mathrm{x}}\right) / \ln \left(T r_{\mathrm{n}}\right)=\mu_{\mathrm{x}} / \mu_{\mathrm{n}} .
$$

The term in thickness is canceled out in the right-hand side of Eq. (2) when the same sample is used for $\operatorname{Tr}_{n}$ and $\operatorname{Tr}_{\mathrm{x}}$. According to Eq. (2), the both transmission of neutron and X-ray would be lead to the ratio of the linear attenuation coefficients. To test the above relation, the transmissions of various samples for the neutron and X-ray were calculated with the PHITS code. In the simulation, a sample with a diameter of $1 \mathrm{~cm}$ and a thickness of 0.1 or $1 \mathrm{~mm}$ was defined within a vacuum space. A single element which was composed of stable isotopes with natural abundances [12] was set as the material of sample except gas. A monochromatic neutron or X-ray source was a pencil-like beam in 1-cm diameter, where the energies of neutron and X-ray were $25 \mathrm{meV}$ and $200 \mathrm{keV}$, respectively. The transmitted neutrons (or X-rays) were counted by a tally that was located at the distance of $100 \mathrm{~cm}$ from the sample, where the area of tally was $1 \mathrm{~cm}$ in diameter. By substituting the calculated transmissions to Eq. (2), the $R_{x n}$ was obtained for each sample. In Fig. 5, the $R_{\mathrm{xn}}$ are plotted against the atomic numbers of various samples. The inverse triangle and square indicate the thickness of sample $t=0.1$ and $1 \mathrm{~mm}$, respectively, where the result for the Gd sample with $t=1 \mathrm{~mm}$ is not plotted because the neutron transmission is practically zero. The simulation results for different thicknesses are in good agreement as shown in Fig. 5. Accordingly, the use of $R_{\mathrm{x} n}$ is expected to enhance the information to distinguish the substance of sample in addition to the use of each transmission. Note that the calculated $R_{\mathrm{xn}}$ is directly affected by the uncertainties of attenuation coefficients especially nuclear data as shown in Eq. (2). While the substances for the almost same $R_{\mathrm{xn}}$ could not be distinguished such as Rb$\mathrm{Pd}$ region in Fig. 5, the some possible substances could be limited using the $R_{\mathrm{xn}}$.

\section{Conclusion}

By using the composite source system at Hokkaido University, the transmission images were measured using the neutron and X-ray beams without change of a sample and detector setup. In the measurement, the detector based on the vacuum-tube type neutron color image intensifier was applied to the beams for dual-purpose, where the digital camera was used as an image recorder. The sample was a flashlight involving a rechargeable cell. The measured transmission images were directly compared the transmission ratio of the neutron and X-ray transmission at each pixel. As the result, an enhanced contrast image obtained from the dataset. In addition, the test measurements using the Gd-type neutron grid were performed with the cold neutron source at Hokkaido University. The grid effectively reduced the background neutrons that were scattered from the sample. On the other hand, the simulations of the neutron and
X-ray transmissions for various substances were performed using the PHITS code. By Eq. (2), the $R_{\mathrm{xn}}$ was obtained from the transmissions for the $25-\mathrm{meV}$ neutron and $200-\mathrm{keV}$ X-ray beam. For the identical substance, the values of $R_{x n}$ were consistent between the samples with the thickness of 0.1 and $1 \mathrm{~mm}$. The $R_{\mathrm{xn}}$ depend little on the thickness of sample in contrast with the transmissions of neutron and X-ray. It is expected that the $R_{\mathrm{xn}}$ give additional information to distinguish the substance of sample, where the point of the complementary imaging with the $R_{\mathrm{xn}}$ is to measure both transmissions of neutron and X-ray without change of a sample and detector setup. In future, we have a plan of test measurements of neutron and X-ray transmissions for a sample using the NCII combined with the high-speed camera in order to estimate the substance from the measured $R_{\mathrm{xn}}$ through the calculated $R_{x n}$.

The work was supported under the Photon and Quantum Basic Research Coordinated Development Program by the Ministry of Education, Culture, Sports, Science, and Technology (MEXT), Japan.

\section{References}

[1] K.Y. Hara, H. Taira, H. Sato, and T. Kamiyama, Proceedings of the 2014 Symposium on Nuclear Data, November 27-28, 2014, Sapporo, JAEA-Conf 2015-003, p. 203

[2] T. Kamiyama, K.Y. Hara, H. Taira, and H. Sato, Nuovo Ciemento 38 C, 187 (2015)

[3] K.Y. Hara, H. Taira, H. Sato, and T. Kamiyama, Proceedings of the 2015 Symposium on Nuclear Data, November 19-20, 2015, Tokai-mura, Ibaraki, JAEA-Conf 2016-004, p. 199

[4] T. Sato, K. Niita, N. Matsuda, S. Hashimoto, Y. Iwamoto, S. Noda, T. Ogawa, H. Iwase, H. Nakashima, T. Fukahori, K. Okumura, T. Kai, S. Chiba, T. Furuta, L. Sihver, J. Nucl. Sci. Technol. 50, 913 (2013)

[5] K. Nittoh, C. Konagai, M. Yahagi, Y. Kiyanagi and T. Kamiyama, Phys. Proc. 69, 177 (2015)

[6] K.Y. Hara, H. Harada, Y. Toh, and J. Hori, Nucl. Instrum. Methods A 723, 121 (2013)

[7] Coffin D. DCRAW. Available from: http://www . cybercom.net/ dcoffin/dcraw/

[8] H. Sato, K. Watanabe, K. Kiyokawa, R. Kiyanagi, K. Y. Hara, T. Kamiyama, M. Furusaka, T. Shinohara and Y. Kiyanagi, Proceedings of the 8th International Topical Meeting on Neutron Radiography, September 4-8, 2016, Beijing (to be published)

[9] Y. Kiyanagi, Nucl. Instrum. Methods 562, 561 (2006)

[10] S. Uno, T. Uchida, M. Sekimoto, T. Murakami, K. Miyama, M. Shoji, E. Nakano, T. Koike, K. Morita, H. Satoh, T. Kamiyama, Y. Kiyanagi, Phys. Proc. 26, 142 (2012)

[11] K. Shibata, O. Iwamoto, T. Nakagawa, N. Iwamoto, A. Ichihara, S. Kunieda, S. Chiba, K. Furutaka, N. Otuka, T. Ohsawa, T. Murata, H. Matsunobu, A. Zukeran, S. Kamada, J. Katakura, J. Nucl Sci Technol. 48, 1 (2011)

[12] M. Berglund and M.E. Wieser, Isotopic compositions of the elements 2009 (IUPAC Technincal Report), Pure Appl. Chem. 83, 397 (2011) 